\title{
Systematic Review on The Effectiveness of Using Carbon Monoxide Measurement in Smoking Cessation Intervention
}

\author{
Muhammad Adil Zainal Abidin, Hayati Kadir @ Shahar, Rosliza Abdul Manaf \\ ${ }^{1}$ Department of Community Health, Faculty of Medicine and Health Sciences, \\ Universiti Putra Malaysia, Serdang Selangor
}

Introduction: Carbon monoxide measurement is one of the biomedical risk assessments in smoking cessation. With the advancement in medical devices, risk assessment among smoker using carbon monoxide can be an effective intervention for quit smoking. A systematic review was conducted on the effectiveness of using carbon monoxide measurement in smoking cessation intervention. Material and methods: A systematic review and narrative synthesis were done using PRISMA guideline. A broad database search on English literature was conducted including Medline, PubMed, Scopus and Science Direct from 2008 till 2017. Additional studies were also searched through snowballing technique by searching the bibliographies of selected studies. Five studies met the criteria with two studies were done in clinical settings while the other was done at population settings. The studies were conducted in the United States of America, United Kingdom and South Korea. All study were two-arm randomized controlled trials with variation in the outcome measurement mainly intention to quit, quit attempt and smoking abstinence. There was also variation in the duration of follow up ranging from 1 week to 12 months. Results: Only three studies found statistically significant increased in motivation or intention to quit smoking but only at 1 month follow up. All five study found no significant difference in frequency of quit attempt or smoking abstinence. Most of the studies recruited participants based on voluntary participation thus limiting the generalizability of the results. Conclusions: The present review has found limited studies on the effect of carbon monoxide measurement intervention for quit smoking. It may only be beneficial at the motivational level and not translated into behavioural changes. However, the effect too was short term and not sustained in the long term. 